\title{
Error Analysis of Radial Motion Measurement of Ultra-Precision Spindle*
}

\author{
Risheng Zhang1, Jialin Yang1\#, Erwei Shang², Yanqiu Chen², Yu Liu² \\ ${ }^{1}$ Institute of Machinery Manufacturing Technology, China Academy of Engineering Physics, Mianyang, China \\ ${ }^{2}$ School of Mechanical Engineering, Jiangnan University, Wuxi, China \\ Email: "261164382@qq.com
}

How to cite this paper: Zhang, R.S., Yang, J.L., Shang, E.W., Chen, Y.Q. and Liu, Y. (2018) Error Analysis of Radial Motion Measurement of Ultra-Precision Spindle. World Journal of Engineering and Technology, 6, 567-574.

https://doi.org/10.4236/wjet.2018.63034

Received: June 1, 2018

Accepted: July 20, 2018

Published: July 23, 2018

Copyright $\odot 2018$ by authors and Scientific Research Publishing Inc. This work is licensed under the Creative Commons Attribution International License (CC BY 4.0).

http://creativecommons.org/licenses/by/4.0/

\begin{abstract}
This paper gives an error analysis of radial motion measurement of ultra-precision spindle including nonlinearity error of capacitive displacement probes, misalignment error of probes, eccentric error of artifact ball and error induced by different error separating methods. Firstly, nonlinearity of a capacitive displacement probe targeting a spherical surface is investigated through experiment and the phenomena of fake displacement induced by lateral offset of the probe relative to an artifact ball are discussed. It is shown that the error motion in radial and axial direction and eccentric rotation of artifact ball will both induce lateral offset which causes a fake output of probes. Moreover, measurement error induced by angular positioning error for three famous error separating methods is detailed.
\end{abstract}

\section{Keywords}

Error Motion, Spindle Metrology, Ultra-Precision Spindle, Error Analysis

\section{Introduction}

Ultra-precision spindle or rotating table usually working on aerostatic or hydrostatic principle plays an important role in ultra-precision machine tools. The rotational accuracy of spindle is a main factor influencing the machining accuracy of ultra-precision machine tool [1]. Traditional method no longer applies to error motion measurement for ultra-precision axis because of artifact form error. As a result, several error separating methods have been developed. The most well-known methods such as Donaldson reversal, multi-steps and multi-probe have been demonstrated to approach uncertainty on order of nanometers [2] [3]

*Supported by National Science and Technology Major Project of High-level Numerical Controlling Machine Tools and Basic Manufacturing Equipment with the granted No. 2017ZX04011001. 
[4]. Grejda proposed a modified reversal method to eliminate mounting error induced by misalignment of artifact and probe by relocating the spindle stator using a rotary table instead [5]. However, none of the above researches made a comprehensive investigation into error analysis considering all the factors such as alignment error, error separating methods, probe nonlinearity. Moreover, nonlinearity of capacitive probe when targeting spherical artifact is not taken into consideration in detail. Especially, nonlinearity when a probe moving laterally relative to a spherical surface has not been investigated ever before. Although $\mathrm{R}$. Ryan Vallance studied nonlinearity when a probe moving axially relative to a spherical surface [6].

\section{Error Analysis}

\subsection{Capacitive Probe Nonlinearity Targeting Ball Surface}

In order to study nonlinearity of a capacitive probe moving laterally relative to a spherical surface, an experiment is conducted shown in Figure 1(a) where the probe moves laterally every time by some micrometers while a same probe is used to measure the lateral displacement. The data is shown in Figure 1(b) and a quadratic curve is used to fit the experimental data. It is obvious that the nonlinearity exists when the probe moves laterally above the spherical surface. This leads to two problems when measuring radial error of axis of rotation. One is whether the linear gain will change when the probe targeting spherical surface at different lateral offsets. The other is the lateral component of eccentric movement of artifact ball may lead to additional reading error of a capacitive probe which will be discussed later in section 2.2. To investigate the former problem one experiment is made in which readings of a probe approaching the artifact ball at different lateral offsets and the results are shown in Figure 1(c). It is concluded that the linear gain remains constant when lateral displacement varying between 0 and $40 \mu \mathrm{m}$. When measure error motion, the lateral displacement is always keep minimum by adjusting the probe in lateral direction to approach the highest point of the artifact ball and this will ensure the linear gain is constant.

\subsection{Eccentricity Induced Lateral Misalignment}

A misalignment between the artifact and axis of rotation leads to eccentric error in the probe signals. Two primary methods exist to eliminate this effect, such as the least quadratic circle and the Fourier analysis to remove the fundamental frequency. However, little attention is given to the fact that lateral component of eccentric movement vector of artifact ball may lead to additional reading error of capacitive probe. Set the eccentric error to be $e$. At angular position $\theta$, the lateral and the radial components of eccentric error are $e * \cos \theta$ and $e * \sin \theta$ respectively. Assuming the initial lateral offset of the probe $e_{0}$ relative to the ball is shown in Figure 2.

According to 2.1, output of the probe $e_{\text {lateral_effect }}$ caused by lateral offset $\chi$ can be presented by 


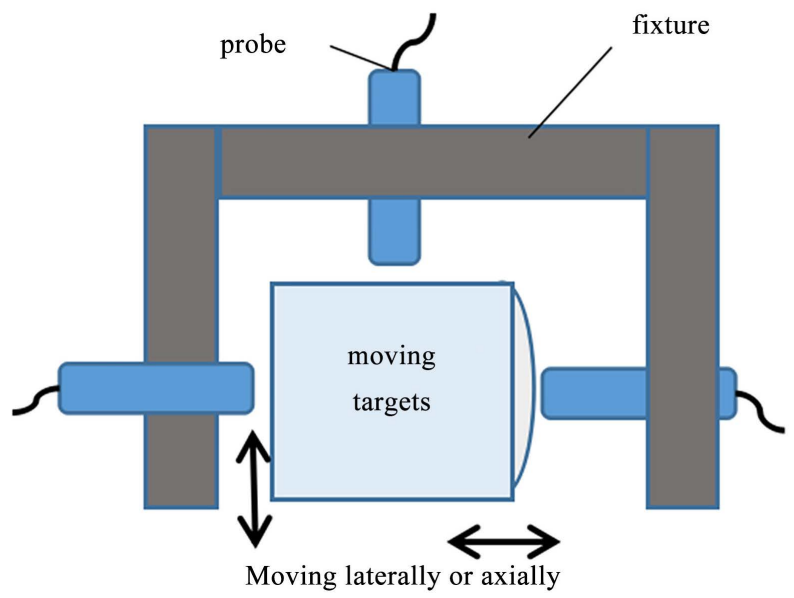

(a)

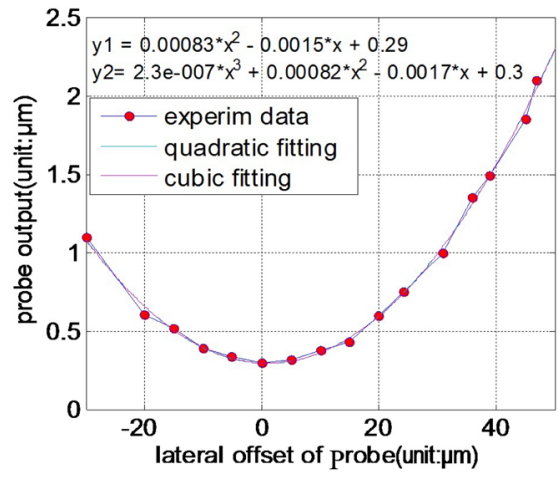

(b)

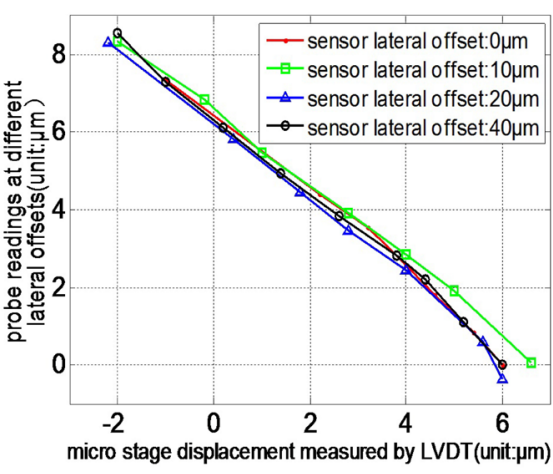

(c)

Figure 1. Nonlinearity of a capacitive sensor induced by lateral offset relative to a spherical artifact. (a) Experiment setup; (b) Capacitive probe output when moving laterally at different offset distances. y1 and y2 are quadratic and cubic fitting equations respectively; (c) Experiment data to identify the output characteristic of capacitive probe at different lateral offset distances.

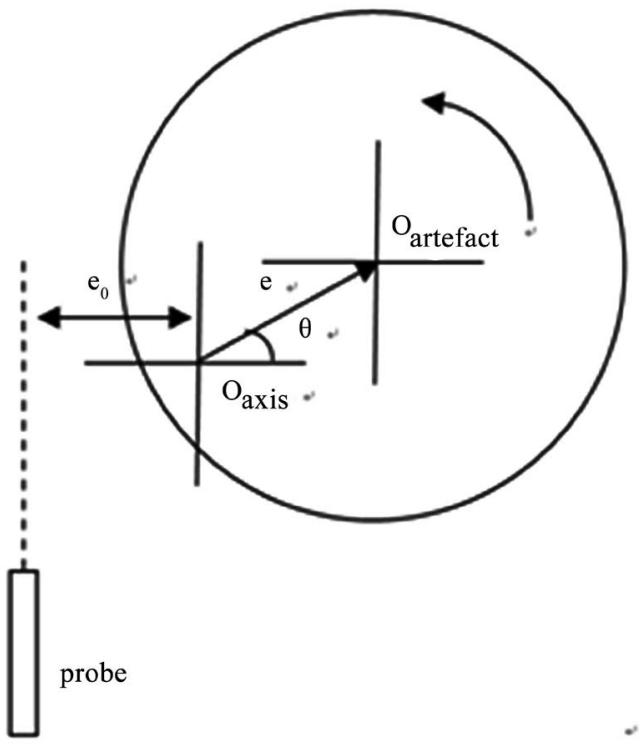

Figure 2. Eccentric error induced lateral offset. 


$$
e_{\text {lateral_effect }}=a \chi^{2}=a\left(e_{0}+e \cos \theta\right)^{2}
$$

where, $\alpha$-the identified coefficient and in this paper $\mathrm{a}=0.00082 \mu \mathrm{m}^{-2}$.

The total contribution to the probe output caused by eccentric error is expressed by

$$
\begin{aligned}
& e_{\text {eccentric_effect }} \\
& =e_{\text {lateral_effect }}+e \sin \theta \\
& =\alpha\left(e_{0}+e \cos \theta\right)^{2}+e \sin \theta \\
& =\alpha e_{0}^{2}+\frac{1}{2} \alpha e^{2}+\frac{1}{2} \alpha e^{2} \cos 2 \theta+2 \alpha e_{0} e \cos \theta+e \sin \theta
\end{aligned}
$$

From this formula, second order and first order errors will be included in the probe output and when the eccentric error $\mathrm{e}=5 \mu \mathrm{m}$ the second order error will be up to $10 \mathrm{~nm}$ which will be an unacceptable error and be impossible to be eliminated by mathematical method. The last two 1st order components in this formula can be removed by Fourier analysis to remove the fundamental frequency.

\subsection{Misalignment Error of Probe: Tilt Error}

When considering radial error motion, one of the important error sources is attributed to misalignment between the capacitive probe and the artifact ball as is shown in Figure 3. The probe output is affected by four error motion components of which two are in the error sensitive direction and the other two in the error insensitive direction. The components have the following relationship:

$$
\begin{gathered}
\boldsymbol{e}_{a}=\boldsymbol{e}_{a n}+\boldsymbol{e}_{a t} \\
\boldsymbol{e}_{r}=\boldsymbol{e}_{r n}+\boldsymbol{e}_{r t}
\end{gathered}
$$

where, $\boldsymbol{e}_{a t}$ and $\boldsymbol{e}_{r t}$ are error motion components in the error sensitive direcion, $\boldsymbol{e}_{r n}$ and $\boldsymbol{e}_{a n}$ in the error insensitive direction, and $\boldsymbol{e}_{a}$ is the axial error motion and $\boldsymbol{e}_{r}$ the radial error motion. Accordingly, the output $m_{1}$ of probe can be expressed as the combination effects of two parts, namely

$$
m_{1}=S_{x}+E
$$

where $S_{x}$ and $\mathrm{E}$ are radial error motion in $\mathrm{X}$ direction and the error induced by misalignment, respectively. We have

$$
E=e_{r n}+e_{a t}+f\left(e_{a n}+e_{r t}\right)-e_{r}
$$

Substituting $e_{r n}=e_{r} \cos \varphi$ and $e_{a t}=e_{a} \sin \varphi$ in to (6) yields

$$
E=e_{r}(\cos \varphi-1)+e_{a} \sin \varphi+f\left(e_{a n}+e_{r t}\right)
$$

where function $f(\cdot)$ corresponds to the lateral offset effects which is detailed in section 2.1 and $\varphi$ is the tilt angle. Considering the lateral offset $e_{a n}$ and $e_{r t}$ are much smaller relative to the initial distance $e_{0}$, we have

$$
\begin{gathered}
f\left(e_{a n}+e_{r t}\right)=a\left(e_{0}+e_{a n}+e_{r t}\right)^{2}-a e_{0}^{2} \\
f\left(e_{a n}+e_{r t}\right) \approx 2 a e_{0}\left(e_{a n}+e_{r t}\right)
\end{gathered}
$$




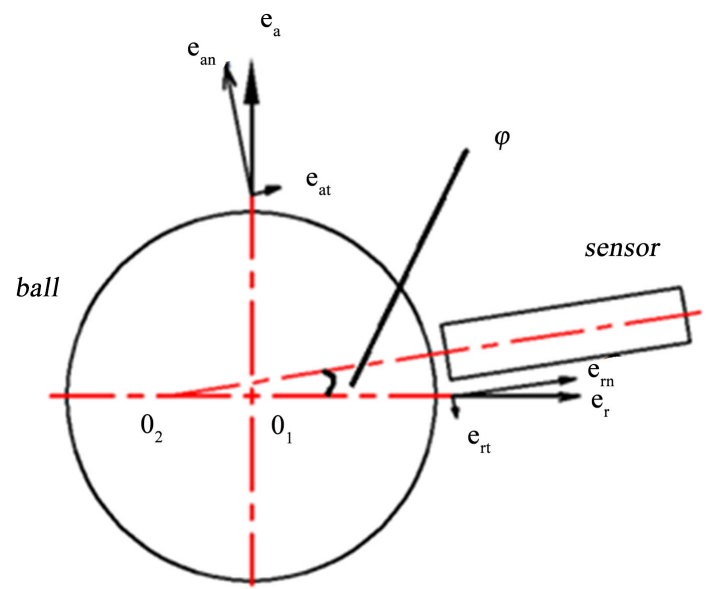

Figure 3. General misalignment between the probe and the artifact.

when $\varphi$ is small enough, we have

$$
E \approx e_{a} \varphi+2 a e_{0}\left(e_{a n}+e_{r t}\right)
$$

It can be concluded from (2) and (10) that the error motion in radial and axial direction and eccentric rotation of artifact ball will both induce lateral offset which causes a fake output of probes. When axial error motion is $0.4 \mu \mathrm{m}$ and the initial lateral offset $e_{0}$ is $20 \mu \mathrm{m}$, the maximum error due to lateral offset effects is up to $13 \mathrm{~nm}$, which is a large measurement error in calibration of an ultra-precision spindle.

\section{Positioning Error of Different Error Separations Methods}

\subsection{Donaldson Reversal Method}

Let the angular positioning error of artifact after reversal be $\varphi$ which is shown in Figure 4(a), then the measurement error induced by angular error after reversing the artifact is derived as

$$
E(\theta)=\frac{R(\theta)-R(\theta+\varphi)}{2} \approx-\frac{\varphi}{2} R^{\prime}(\theta)
$$

where $R(\theta)$ is roundness of the artifact.

The Donaldson reversal method needs to rotate the probe by 180 degrees relative to the rotor of the spindle measured at the same time. Angular position error of the probe will be introduced into the measurement signal. This kind of error is illustrated in Figure 4(b). The corresponding measurement error is derived as

$$
\begin{gathered}
M_{2}(\theta)=R(\theta-\varphi)-S_{x}(\theta) \cos \varphi+S_{y}(\theta) \sin \varphi \\
E(\theta)=\frac{M_{1}(\theta)-M_{2}(\theta)}{2}-S_{x}(\theta) \\
E(\theta)=\frac{1}{2}\left[R(\theta)-R(\theta-\varphi)+S_{x}(\cos \varphi-1)-S_{y} \sin \varphi\right]
\end{gathered}
$$




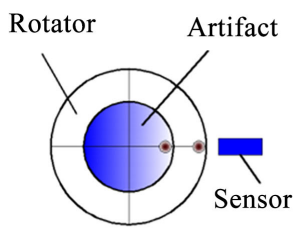

Before reversal

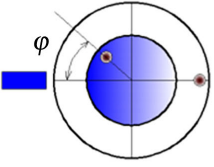

After reversal

(a)

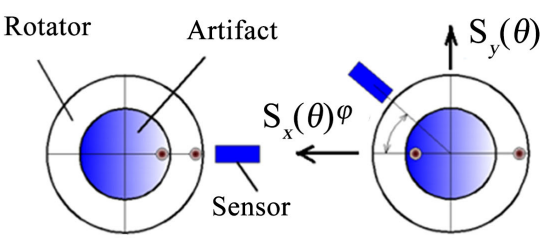

Before reversal
After reversal

(b)

Figure 4. Illustration of positioning error of Donaldson reversal method. (a) Positioning error of artifact ball; (b) Positioning error of probe.

$$
E(\theta) \approx \frac{1}{2}\left[\varphi\left(R^{\prime}(\theta)-S_{y}\right)-\frac{1}{2} S_{x}(\theta) \varphi^{2}+o(\varphi)+o\left(\varphi^{4}\right)\right]
$$

where $S_{x}$ and $S_{y}$ are error motion components in X and Y directions respectively. If $\varphi$ is efficiently small and the measurement error will be simplified as

$$
E(\theta) \approx \frac{1}{2}\left[\varphi\left(R^{\prime}(\theta)-S_{y}\right)+o(\varphi)\right]
$$

\subsection{Multi-Position Method}

When using multi-position method to separate roundness of the artifact and rotating the artifact by a constant angle $\varphi$, an angular error $\Delta_{i}$ exists, as is shown in Figure 5(a). According to the principle introduced in [4], the measurement error induced by angular error $\Delta_{k}$ is derived as

$$
\begin{aligned}
E(\theta) & =\frac{1}{N} \sum_{k=0}^{N-1} R\left(\theta+k \varphi+\Delta_{k}\right) \\
& \approx \frac{1}{N} \sum_{k=0}^{N-1}\left[R(\theta+k \varphi)+R^{\prime}(\theta+k \varphi) \Delta_{k}+o\left(\Delta_{k}\right)\right]
\end{aligned}
$$

As roundness of the artifact can be expressed as Fourier series and when $\mathrm{N}$ is an even integer, we have $\sum_{k=0}^{N-1} R(\theta+k \varphi)=0$. If $\Delta_{k}$ is small enough, we have

$$
E(\theta) \approx \frac{1}{N} \sum_{k=0}^{N-1} R^{\prime}(\theta+k \varphi) \Delta_{k}
$$

\subsection{Multi-Probe Method}

Three-probe method is detailed in [5], here gives only the formulas. Define $M(\theta)$ as linear combination of $m_{1}(\theta), m_{2}(\theta)$ and $m_{3}(\theta)$ with coefficients $\mathrm{a}, \mathrm{b}$ and $\mathrm{c}$ respectively, namely

$$
\left\{\begin{array}{l}
M(\theta)=m_{A}(\theta)+b m_{B}(\theta)+c m_{C}(\theta) \\
b=-\frac{\sin \beta}{[\sin (\beta-\alpha)]} \\
c=\frac{\sin \alpha}{[\sin (\beta-\alpha)]}
\end{array}\right.
$$

where $m_{1}(\theta), m_{2}(\theta)$ and $m_{3}(\theta)$ are outputs of sensors, and 


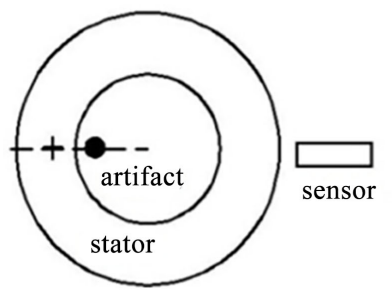

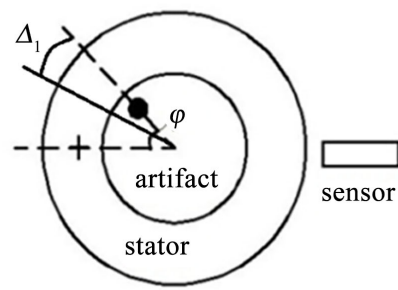

(a)
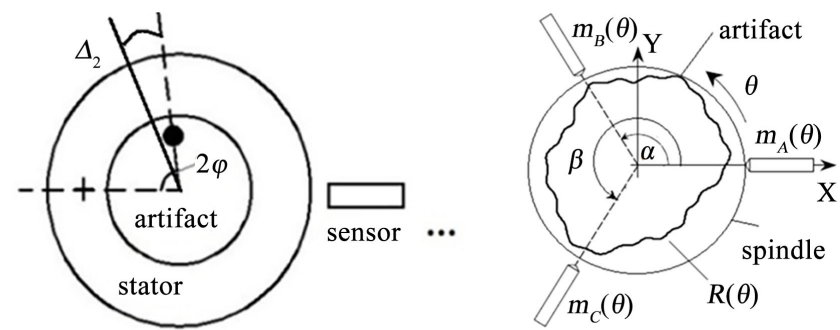

(b)

Figure 5. Angular positioning error of multi-position (a) and three-probe methods (b).

$b=-\sin \beta /[\sin (\beta-\alpha)], \quad c=\sin \alpha /[\sin (\beta-\alpha)]$. Applying discrete Fourier transformation (DFT) to formula (19) yields

$$
M(k)=\left(1+b \mathrm{e}^{-j k \alpha}+c \mathrm{e}^{-j k \beta}\right) R(k)
$$

when three-probe method is used, let angular position errors of probes at positions $\alpha$ and $\beta$ be $\delta \alpha$ and $\delta \beta$ respectively, as is shown in Figure 5(b). We have the measurement error of roundness of artifact in frequent domain

$$
E(k)=\left(\frac{1}{W_{1}}-\frac{1}{W}\right) M(k)-\frac{1}{W_{1}}\left[C_{1} S_{x}(k)+C_{2} S_{y}(k)\right]
$$

where $W_{1}(k)=1+b e^{-j k(\alpha+\delta \alpha)}+c e^{-j k(\beta+\delta \beta)}, W(k)=1+b e^{-j k \alpha}+c e^{-j k \beta}$, $C_{1}=1+\mathrm{b} \cos (\alpha+\delta \alpha)+\cos (\beta+\delta \beta), \quad C_{2}=\mathrm{b} \sin (\alpha+\delta \alpha)+\operatorname{csin}(\beta+\delta \beta) \quad$ By inverse Fouries transformation we have the measurement error $e(\theta)=\operatorname{IDFT}(E(k))$.

\section{Summary}

Factors influencing measurement error of radial error motion are discussed in detail. Nonlinearity of a capacitive displacement probe targeting a spherical surface is investigated through experiment and the phenomena of fake displacement induced by lateral offset of the probe relative to an artifact ball are discussed. It is shown that the error motion in radial and axial direction and eccentric rotation of artifact ball will both induce lateral offset which causes a fake output of probes.

\section{References}

[1] Tauhiduzzaman, M. (2015) Form Error in Diamond Turning. Precision Engineering, 42, 22-36. https://doi.org/10.1016/j.precisioneng.2015.03.006

[2] Evans, C.J., Hocken, R.J. and Estler, W.T. (1996) Self-Calibration: Reversal, Redundancy, Error Separation, and Absolute Testing. CIRP Annals-Manufacturing Technology, 45, 617-634. https://doi.org/10.1016/S0007-8506(07)60515-0

[3] Zhang, G.X., Zhang, Y.H., Yang, S.M., et al. (1997) A Multipoint Method for Spindle Error Motion Measurement. CIRP Annals Manufacturing Technology, 46, 441-445. https://doi.org/10.1016/S0007-8506(07)60861-0

[4] Marsh, E., Couey, J. and Vallance, R. (2006) Nanometer-Level Comparison of Three Spindle Error Motion Separation Techniques. Journal of Manufacturing Science 
and Engineering, 128, 180-187. https://doi.org/10.1115/1.2118747

[5] Grejda, R., Marsh, E. and Vallance, R. (2005) Techniques for Calibrating Spindles with Nanometer Error Motion. Precision Engineering, 29, 113-123.

https://doi.org/10.1016/j.precisioneng.2004.05.003

[6] Vallance, R.R., Marsh, E.R. and Smith, P.T. (2004) Effects of Spherical Targets on Capacitive Displacement Measurements. Journal of Manufacturing Science \& Engineering, 126, 822-829. https://doi.org/10.1115/1.1813476 